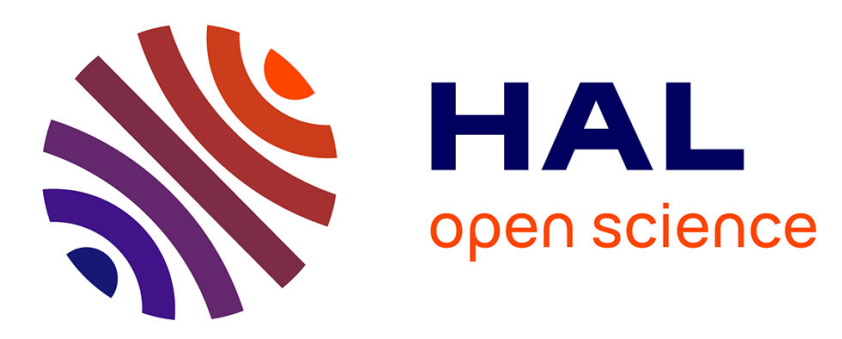

\title{
Influence of Spring Characteristics on the Behavior of Tensegrity Mechanisms
}

Quentin Boehler, Marc Vedrines, Salih Abdelaziz, Philippe Poignet, Pierre

Renaud

\section{- To cite this version:}

Quentin Boehler, Marc Vedrines, Salih Abdelaziz, Philippe Poignet, Pierre Renaud. Influence of Spring Characteristics on the Behavior of Tensegrity Mechanisms. Advances in Robot Kinematics, pp.161-169, 2014, 978-3-319-06698-1. 10.1007/978-3-319-06698-1_18 . lirmm-01018866

\section{HAL Id: lirmm-01018866 \\ https://hal-lirmm.ccsd.cnrs.fr/lirmm-01018866}

Submitted on 8 Jul 2014

HAL is a multi-disciplinary open access archive for the deposit and dissemination of scientific research documents, whether they are published or not. The documents may come from teaching and research institutions in France or abroad, or from public or private research centers.
L'archive ouverte pluridisciplinaire HAL, est destinée au dépôt et à la diffusion de documents scientifiques de niveau recherche, publiés ou non, émanant des établissements d'enseignement et de recherche français ou étrangers, des laboratoires publics ou privés. 


\title{
Influence of spring characteristics on the behavior of tensegrity mechanisms
}

\author{
Quentin Boehler, Marc Vedrines, Salih Abdelaziz, Philippe Poignet, and Pierre
} Renaud

\begin{abstract}
There is today a growing interest for tensegrity mechanisms. Their analysis is however challenging because of their self-stress state. The most popular tensegrity mechanisms use linear springs as tensioned elements. Their synthesis for given user requirements is an open issue. In this article, we propose as a first step to better understand the influence of the spring characteristics, that constitute important design parameters. The influence of spring free length is in particular assessed, considering two planar tensegrity mechanisms. Impact of the spring selection on the workspace, the stiffness and the actuation requirements is observed. The simulation results outline that using nonzero free length springs can be of interest, and conclusions are given on further steps towards a synthesis method.
\end{abstract}

Key words: tensegrity mechanisms, stiffness computation, workspace estimation.

\section{Introduction and scope of the study}

Tensegrity systems can be defined [5] as systems in stable self-equilibrated state comprising a discontinuous set of compressed components, i.e. struts, inside a continuum of tensioned components, either cables or springs. Thanks to the self-stress state, prestress can be imposed for a same topology in order to modify the level of internal forces. A high stiffness-to-mass ratio can thus be reached. Moreover, since the components are axially loaded, they can be very light and so does the system [7]. Tensegrity mechanisms use actuators to modify their configuration $[3,6]$. The tensioned components are then generally linear springs, in order to systematically reach

Quentin Boehler (quentin.boehler@icube.unistra.fr), Marc Vedrines, Pierre Renaud

ICube, France

Salih Abdelaziz, Philippe Poignet

LIRMM, France 
equilibrated configurations without tension loss. They have recently received a lot of attention $[8,9,11]$, and could be interesting solutions for instance in surgical contexts, where lightweight devices with large workspaces are required [1].

The analysis of tensegrity mechanisms is delicate compared to rigid-body mechanisms. The determination of a mechanism configuration requires for instance to take into account joint variables and external loads while respecting self-equilibrium state conditions. The corresponding static model is obtained using so-called form finding methods [10]. Up to now, related works have been focused on the analysis of tensegrity mechanisms, but not yet on their synthesis. Even the relationship between the design parameters and the mechanism behavior, and the estimation of actuator performances for given specifications remains to be investigated. In this paper, we wish therefore to analyze the sensitivity of the tensegrity characteristics to their design parameters.

The spring characteristics strongly influence the mechanism behavior. We therefore concentrate our efforts on the parameters related to the linear springs used as tensioned components. The influence of spring stiffness is here considered, and more importantly the free length of the springs. To our knowledge, estimating the influence of nonzero free lengths on mechanism performances has not yet been covered in the literature.

As a result, we introduce in this paper an analysis of spring stiffness and free length on the behavior of tensegrity mechanisms. Two planar tensegrity mechanisms of Snelson-cross type [2,3] are considered, that respectively exhibit 1 and 2-DOF. In section 2, the 1-DOF mechanism is considered. The mechanism is simple enough to allow a detailed analysis of free length influence on mechanism workspace, stiffness and criteria related to actuator requirements, namely actuator stroke, force and energy consumption. In section 3, a 2-DOF mechanism is analyzed. This mechanism can be used as a tool holder in a surgical task [1]. Conclusions are finally given with emphasis on further steps towards a generalization for other tensegrity mechanisms.

\section{Sensitivity analysis of a 1-DOF tensegrity mechanism}

\subsection{Mechanism description}

The 1-DOF tensegrity mechanism, based on the Snelson-cross planar tensegrity, is represented in Figure 1. It consists in four nodes $\{A, B, C, D\}$. Two rigid struts of length $L$ connect respectively $A$ with $C$ and $B$ with $D$. Three linear springs are installed on the outside of the mechanism, with same stiffness $k$ and free length $l_{0}$. An actuator is used to modify the distance $\|A B\|=\rho$, with the node $A$ anchored to the base and $B$ constrained to move in a horizontal direction. In such a situation, the mechanism exhibits 1 DOF, with nodes $C$ and $D$ that have equal vertical displacements. The output variable is defined as $y$, the $Y$-coordinate of $C$ and $D$, and $\rho$ is the input variable. For sake of simplicity, a so-called neutral configuration is defined as 
$\|A D\|=\|B C||=\| C D \|=\rho=100 \mathrm{~mm}$ with perpendicular struts. It implies that the struts have a length $L=100 \sqrt{2}=141 \mathrm{~mm}$.
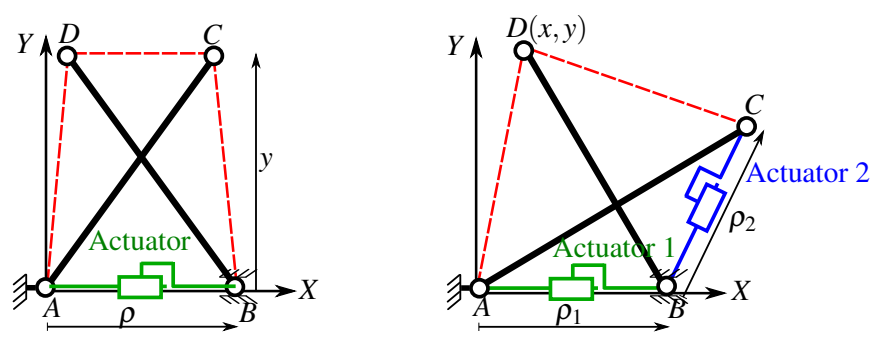

Fig. 1 Kinematic schemes of the 1-DOF (left) and 2-DOF (right) tensegrity mechanisms. In black plain lines, rigid struts and in red dotted lines, linear springs.

\subsection{Modeling and analysis criteria}

The mechanism modeling consists in deriving expressions of the direct and inverse static models. The output variable $y$ is obtained for a given value of $\rho$ by solving the direct static problem. An energy method, as described in [10], is here considered. The mechanism configuration is then obtained by determining the local minimum of the potential energy $U$ stored in the mechanism springs. In our context, $U$ can be easily computed as

$$
U=\frac{1}{2} k\left(2\left(l_{1}-l_{0}\right)^{2}+\left(l_{2}-l_{0}\right)^{2}\right)
$$

with $l_{1}=\|A D\|=\|B C\|$ and $l_{2}=\|C D\|$. The lengths $l_{1}$ and $l_{2}$ can be expressed using simple geometrical relationships as functions of the variables $\rho$ and $y$ and the length $L$. In the following, we are only interested in positive solutions of $y$. The analytic expression of $y=f(\rho)$ is obtained by solving $\frac{\partial U}{\partial y}=0$ for a given $\rho$, using a computer algebra system (Mupad, The Mathworks Inc.). Only one admissible solution is found with the simulated conditions where $\rho \in[70,130] \mathrm{mm}$ and $l_{0} \in[20,80]$ $\mathrm{mm}$. The inverse static model that expresses $\rho$ as a function of $y$ is difficult to obtain if nonzero free length of the springs are considered. As noticed previously in [4], the use of nonzero free length springs in a tensegrity mechanism significantly increases its analysis complexity. In our situation, no analytical expression could be found. A numerical approach is then adopted, with a Levenberg-Marquardt algorithm to compute the $\rho$ value for a given $y$ as the solution of $(y-f(\rho))^{2}=0$. The tensegrity mechanism behavior is assessed by using two types of criteria. The first type corresponds to usual mechanism properties, namely the workspace and the stiffness. The workspace is the range of $y$ values for a given range of $\rho$. The stiffness of the mechanism is defined as $K_{y}=\frac{\delta F_{y}}{\delta u_{y}}$ with $\delta u_{y}$ the infinitesimal displacement of nodes $C$ and $D$ along the $Y$-axis when an infinitesimal external vertical force $\delta F_{y}$ is evenly 
applied on these nodes, while the actuator is locked. As stated in [2], the stiffness $K_{y}$ can be estimated with $\frac{\partial^{2} U}{\partial y^{2}}$. The other type of criteria characterizes the actuation requirements of the mechanism. For a predefined path followed by the mechanism in quasistatic conditions, we compute

- $\Delta \rho$ : the corresponding required actuator stroke

- $\bar{F}$ and $F_{\text {max }}$ : respectively the mean and maximum forces delivered by the actuator

- $\Delta U$ : the variation of potential energy.

Meanwhile, the mean value $\overline{K_{y}}$ and the variation $\Delta K_{y}$ of the stiffness are also estimated. In the presented results, the path is defined as symmetric with respect to the neutral configuration, with a $20-\mathrm{mm}$ displacement. It is discretized in 11 steps to compute the analysis criteria.

\subsection{Results and discussion}

As it can be observed in Eq. (1), the configuration corresponding to the local minimum of the potential energy $U$ does not depend on the springs stiffness $k$. The equilibrium configuration is therefore invariant with respect to $k$. Consequently, modifying the spring stiffness does not modify the mechanism workspace. The mechanism stiffness $K_{y}$ is proportional to $k$ as it can be observed in Eq. (1). The energy variation $\Delta U$ and the actuator force $F$ are also proportional to $k$. The energy variation corresponds to the energy to be delivered by the actuator. The actuation requirements are therefore linearly dependent on the spring stiffness for a given workspace.

The spring free length $l_{0}$ has on the contrary a non-linear influence on the analysis criteria. Figure 2 represents the computation of $y$ and $K_{y}$ according to $l_{0}$ when $\rho$ varies between 70 and $130 \mathrm{~mm}$ in 25 steps, for $k=4 \mathrm{~N} / \mathrm{mm}$. In this example, one may observe that the neutral configuration is not dependent on the spring free length. For a given $y$, increasing $l_{0}$ leads to an increase of $K_{y}$ while the force delivered by the actuator decreases. This may appear unintuitive, but it is explained by the reconfiguration of the mechanism when $l_{0}$ is increased (bottom of Figure 2). The workspace is on the contrary reduced for the same actuation stroke. For given mechanism specifications, interesting compromises therefore exist for nonzero free lengths.

Figure 3 shows the evolution of the criteria related to the actuator requirements. Increasing $l_{0}$ leads to the increase of the actuation stroke $\Delta \rho$ while $\bar{F}, F_{\max }$ are reduced, as well as the potential energy variation $\Delta U$. An optimal value of $l_{0}$ could be searched for in order to adapt the mechanism and the actuator force/displacement characteristics. The influence of the free length on the stiffness is complex, with an increase of the mean value $\overline{K_{y}}$ but a reduction of the variation $\Delta K_{y}$. As a conclusion of this case, the analysis suggests first that nonzero free lengths can be of interest for the mechanism performance, and that the choice of $l_{0}$ is a compromise between mechanism properties $\overline{K_{y}}, \Delta K_{y}$ and the actuator requirements $\Delta \rho, \bar{F}, F_{\max }$ and $\Delta U$. 

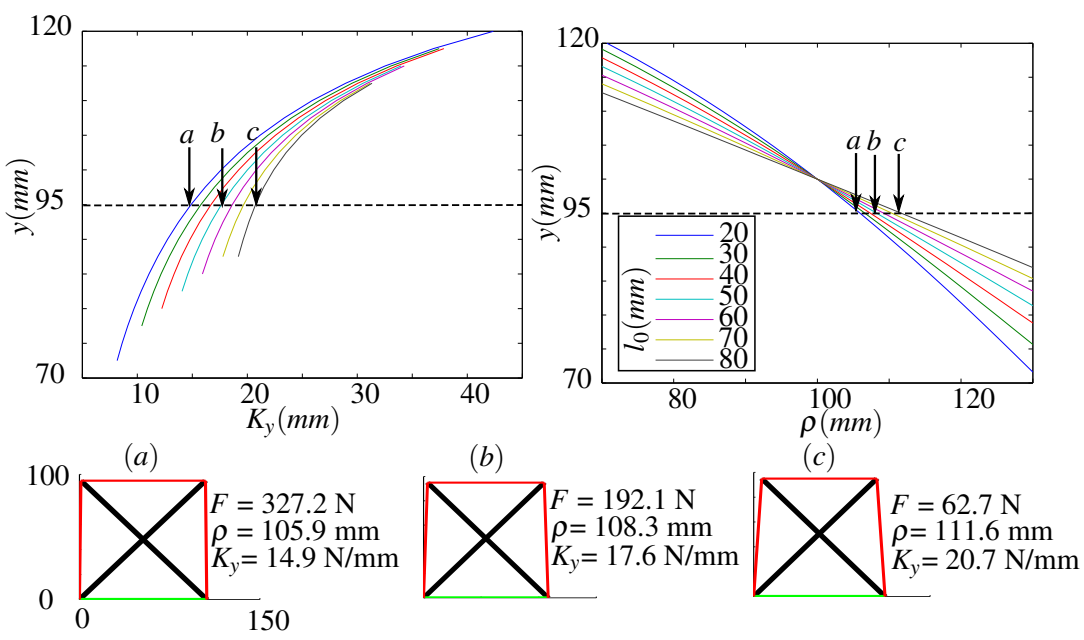

Fig. 2 Relationships between the spring free length $l_{0}$ and the stiffness $K_{y}$ and the workspace. Cases $(a),(b)$ and $(c)$ depict the mechanism at $y=95 \mathrm{~mm}$ for $l_{0}=20,50$ and $80 \mathrm{~mm}$ respectively.
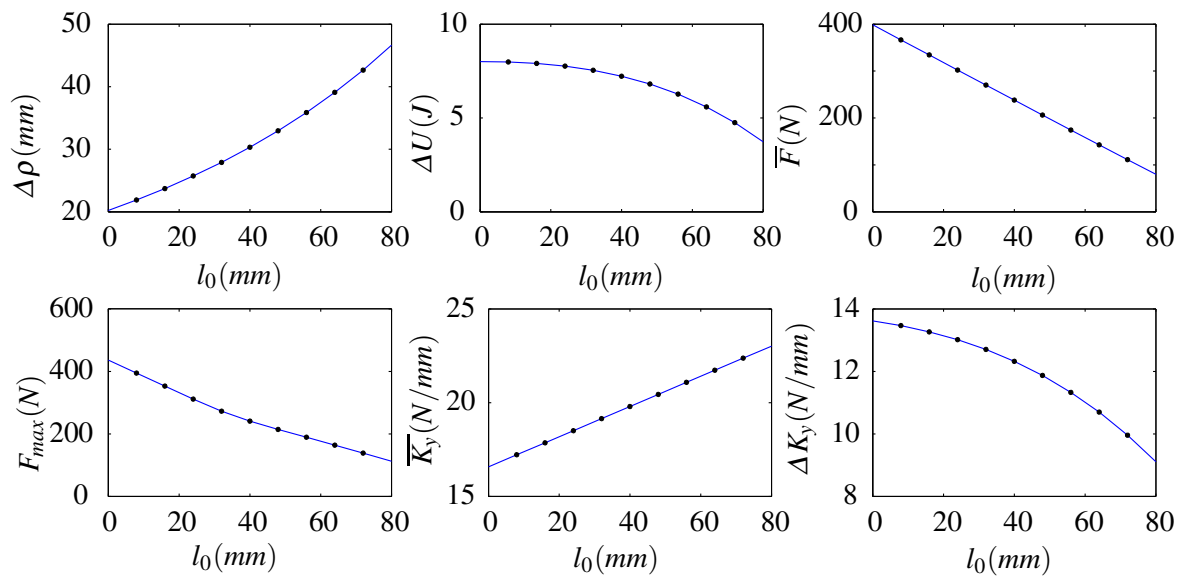

Fig. 3 Evolution of the analysis criteria according to $l_{0}$.

\section{Analysis of a 2-DOF tensegrity mechanism}

\subsection{Mechanism description}

A second actuator is integrated between nodes $B$ and $C$ in the previous tensegrity mechanism (Figure 1). The mechanism becomes a 2-DOF manipulator with two joint variables $\left(\rho_{1}, \rho_{2}\right)$ and its end-effector in $D$. 


\subsection{Modeling and analysis criteria}

For this 2-DOF mechanism, no analytical direct static model could be obtained if springs have a nonzero free length. Hence, the coordinates of $D$ are determined by minimizing the potential energy $U$ using a Nelder-Mead algorithm. The inverse static model is not implemented since it is not necessary for the evaluation.

Contrary to a rigid-body mechanism, the mechanism configuration does not depend only on $\left(\rho_{1}, \rho_{2}\right)$, but also on one parameter that can be either the angle $\widehat{A B D}$, the variable $x$, or $y$. Therefore $U=U_{1}\left(\rho_{1}, \rho_{2}, x\right)=U_{2}\left(\rho_{1}, \rho_{2}, y\right)$, and the end-effector stiffnesses $K_{x}$ and $K_{y}$ in $X$ - and $Y$-direction can be computed as follows

$$
K_{x}=\frac{\partial^{2} U_{1}\left(\rho_{1}, \rho_{2}, x\right)}{\partial x^{2}} \quad \text { (2) } \quad K_{y}=\frac{\partial^{2} U_{2}\left(\rho_{1}, \rho_{2}, y\right)}{\partial y^{2}}
$$

For this mechanism, analysis criteria are related to the mechanism workspace, stiffness and the actuation requirements:

- $A$ is the area of the reachable workspace

- $\overline{K_{x}}$ and $\overline{K_{y}}$ are the mean values of respectively $K_{x}$ and $K_{y}$ over the workspace

- $\Delta K_{x}$ and $\Delta K_{y}$ designate the variations of respectively $K_{x}$ and $K_{y}$ over the workspace

- $\bar{F}$ and $F_{\max }$ are computed as the mean and maximum forces in each actuator.

The workspace is explored with joint ranges that avoid mechanism singularity and loss of tensegrity configuration. A singularity occurs when $\rho_{1}=\rho_{2}=L / 2$, i.e. $B D$ is vertical. The lower bounds of $\rho_{1}$ and $\rho_{2}$ are therefore chosen equal to $75 \mathrm{~mm}$. The strut $B D$ must remain in compression to keep the mechanism in a tensegrity configuration. As a result, the upper bound of the joint variables is such that $\rho_{1}^{2}+\rho_{2}^{2}<5 L^{2} / 2$. A 5 -mm step for each joint variable is considered for the computation. The area $A$ is computed by extracting the edge of the discrete set of positions obtained after the exploration of the joint space. With such joint ranges, and thanks to the mechanism symmetry, $\bar{F}$ and $F_{\max }$ are the same for both actuators.

\subsection{Results and discussion}

Similarly to section 2 , the influence of the spring stiffness $k$ on the analysis criteria is simple. It does not affect the equilibrium configurations, and linearly impacts the required actuators forces and the stiffnesses.

The variation of $K_{y}$ is represented in Figure 4, for a spring stiffness $k=3 \mathrm{~N} / \mathrm{mm}$. For such a mechanism, an end-effector position can sometimes be obtained with two different configurations and hence two different stiffness values. The map is therefore plotted in the joint space. Figure 5 depicts the corresponding reachable workspace. The $l_{0}$ value modifies the shape of the workspace boundaries, and the workspace area. In addition, tension losses can occur that limit the workspace, as it can be observed between the middle and right plots of Figure 5. Further analysis shows that the first tension loss takes place when $D$ is at the middle of $A C$, and 

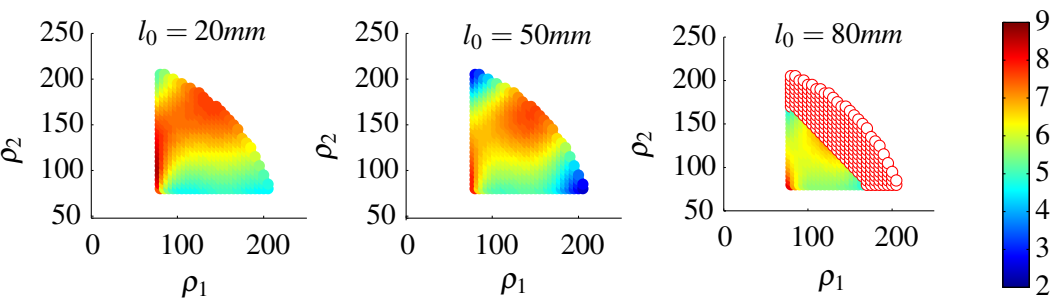

Fig. 4 Variation of the stiffness $K_{y}$ in N/mm in the joint space for $l_{0}=20,50$ and $80 \mathrm{~mm}$. In red circles: unreachable configurations because of tension loss.
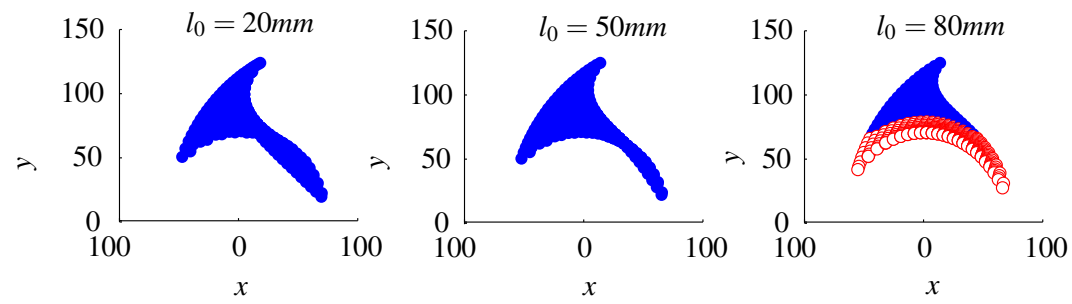

Fig. 5 Reachable workspace for $l_{0}=20,50$ and $80 \mathrm{~mm}$. Blue dots: reachable positions / red circles: unreachable positions because of tension loss.

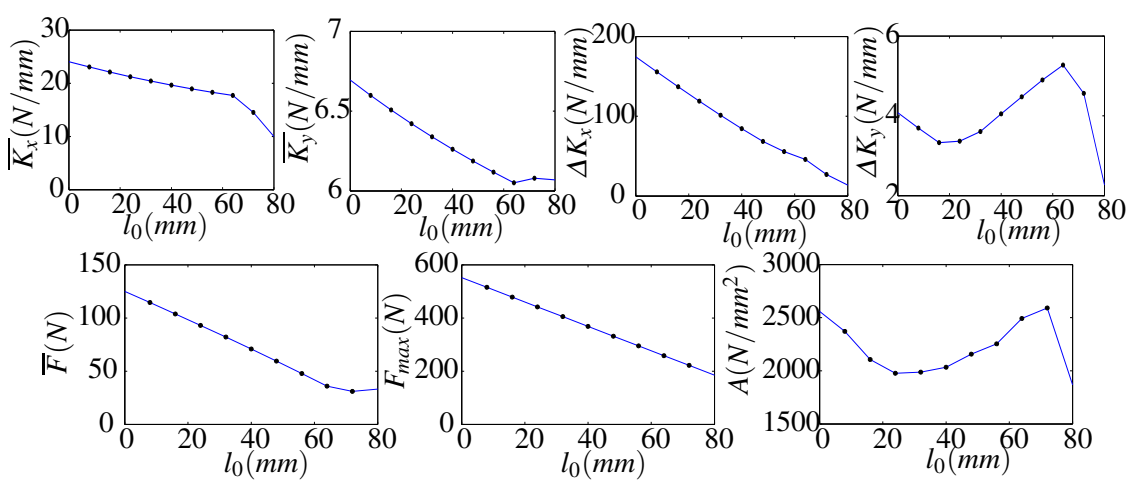

Fig. 6 Evolution of the analysis criteria according to $l_{0}$.

that $l_{0}=l_{0 \text { loss }}=L / 2 \simeq 70 \mathrm{~mm}$. The $l_{0}$ value also affects the mean value and the variation of the stiffness over the workspace (left and middle plots of Figure 4).

Figure 6 shows the evolution of the analysis criteria according to $l_{0}$. This evolution of the criteria is notably affected by choosing $l_{0}>l_{0}$ loss, with in particular a fast reduction of the workspace area $A$. If $l_{0}<l_{0}$ loss $, \overline{K_{x}}, \overline{K_{y}}, \bar{F}$ and $F_{\text {max }}$ decrease when $l_{0}$ increases. Although $\Delta K_{x}$ is also decreasing monotonously with respect to $l_{0}$, the evolution of $\Delta K_{y}$ is more complex (as it can be seen on Figure 4). The lowest value of $\Delta K_{x}$ is indeed obtained for a nonzero value of $l_{0}$. The evolution of the workspace area $A$ is also non uniform. Increasing $l_{0}$ does not necessarily decrease the size of the workspace. 
The criteria sensitivity to the free length value is different from the one observed for the 1-DOF mechanism. Increasing $l_{0}$ can lead to tension losses, which is quite logical, and affects stiffness and workspace properties. However, the free length can be used to lower the required actuator forces and an adequate choice of $l_{0}$ can help increasing the workspace size.

\section{Conclusions}

In this paper, influence of the spring characteristics on tensegrity mechanisms was considered. Using analysis criteria related to the mechanism properties and the actuator requirements, two planar mechanisms were analyzed. The first major remark is that increasing the free length of the springs can have a beneficial impact on the mechanism or the selection of an adequate mechanism. Whereas the spring stiffness influence can be easily assessed, relationships between the selected criteria and the free length is much more complex. The determination of the spring free length during a mechanism synthesis will need to be performed simultaneously with other design parameters related to the mechanism geometry. The observed significant nonlinearities of the sensitivity curves outline also that the selection of the optimization strategy will be delicate, and will be investigated as the next step of this work as well as the generalization for other tensegrity mechanisms.

\section{Acknowledgment}

This work was supported by French state funds managed by the ANR within the Investissements d'Avenir programme (Labex CAMI - ANR-11-LABX-0004) and by the Région Alsace.

\section{References}

1. Abdelaziz, S., Esteveny, L., Renaud, P., Bayle, B., Barbe, L., De Mathelin, M., Gangi, A.: Design considerations for a novel MRI compatible manipulator for prostate cryoablation. Int. J. of Comp. Assist. Rad. and Surg. 6(6), 811-819 (2011)

2. Arsenault, M., Gosselin, C.: Kinematic, static, and dynamic analysis of a planar one-degreeof-freedom tensegrity mechanism. ASME J. of Mech. Des. 127(6), 1152-1160 (2005)

3. Arsenault, M., Gosselin, C.: Kinematic, static and dynamic analysis of a planar 2-DOF tensegrity mechanism. Mechanism and Machine Theory 41(9), 1072-1089 (2006)

4. Crane, C., Bayat, J., Vikas, V., Roberts, R.: Kinematic analysis of a planar tensegrity mechanism with pre-stressed springs. In: Adv. in Rob. Kin.: Analysis and Design, pp. 419-427 (2008)

5. Motro, R.: Tensegrity: Structural Systems for the Future. Elsevier (2003) 
6. Shekarforoush, S., Eghtesad, M., Farid, M.: Kinematic and static analyses of statically balanced spatial tensegrity mechanism with active compliant components. J. of Intell. Robot Syst. 71(3-4), 287-302 (2013)

7. Skelton, R., Oliveira, M.d.: Tensegrity Systems. Springer (2009)

8. Skelton, R., Sultan, C.: Controllable tensegrity: a new class of smart structures. In: Proc. SPIE, Smart Structures and Materials, vol. 3039, pp. 166-177 (1997)

9. Sultan, C., Corless, M., Skelton, R.: Tensegrity flight simulator. Journal of Guidance, Control, and Dynamics 23(6), 1055-1064 (2000)

10. Tibert, A., Pellegrino, S.: Review of form-finding methods for tensegrity structures. Int. J. of Space Struct. 18(4), 209-223 (2003)

11. Tran, T., Crane, C., Duffy, J.: The reverse displacement analysis of a tensegrity based parallel mechanism. In: Proc. of World Autom. Congress, vol. 14, pp. 637-643 (2002) 